\title{
Region-based Image Fusion Method with Dual-Tree Complex Wavelet Transform
}

\author{
Xuanni Zhang ${ }^{1, a}$, Fan $\mathrm{Lu}^{2, \mathrm{~b}}$ \\ ${ }^{1}$ Institute of Physics and Electronic Engineering, Xianyang normal University Xianyang, Xianyang \\ 712000 China \\ ${ }^{2}$ Xi'an longi silicon materials corporation Xi'an, Xi'an 710100 of China \\ amoishel@163.com, blfa@longi-silicon.com
}

\begin{abstract}
Dual-Tree Complex Wavelet Transform is an enhancement to the discrete wavelet transform, with important additional properties: it is nearly shift invariant and directionally selective in two or higher dimensions. And also can overcome the problem of distortion in spectrial and spatial resolution of fused image with other techniques. Combined with region-based fusion method, it can obtain better fusion results. All these had been proved by the experiment.
\end{abstract}

Keywords: Dual-Tree Complex Wavelet Transform; Region-based fusion; Shift invariant; Resolution; Directionally selective.

\section{Introduction}

Multispectral sensors are increasingly being employed in satellite imagery of the earth, multispectral data is required in order to extract the maximum amount of information from a scene, provide the desired fusion image of information into a single picture for rapid assessment [1-4]. All these rely on the image fusion, the combination of the multi-sensor images fully take into account the complementary and supplementary provided by different data sources and optimize the classification of cartographic objects. Thus many image fusion techniques and software have been developed, such as the well-known Principle Component Analyses (PCA), Intensity Hue Saturation (HIS), and Wavelet-based image fusion and so on. One drawback of PCA and HIS is some distortion of spectral characteristics in the original multispectral image [2]. Wavelet-based image fusion can overcome this problem, and provides high spectral quality of the fused satellite images [5], however, it reduces much spatial information, so an improved method has been proposed in this paper that can keep the spectral and spatial resolution that is Dual-Tree Complex Wavelet Transform (DT-CWT).

\section{Region-based Image Fusion Method with Dual-Tree Complex Wavelet Transform}

\subsection{Region-based image fusion with complex wavelets}

DT-CWT is an enhancement to the discrete wavelet transform, with important additional properties: it is nearly shift invariant and directionally selective in two or higher dimensions. The multidimensional DT-CWT is non-separable but is based on a computationally efficient separable filter bank.

The region-based fusion can obtain the best fusion results by considering the nature of points in each region altogether, and use some segmentation algorithm to separate an original image into different regions, and then design different rules for different regions[6-9]. For 2-D image, it can be filtred separately along clumns and then rows by the way like 1-D. To represent fully a real 2-D signal, it must filter with complex conjugates of the column and row filter. Furthermore, it remains computationally efficient, since in fact it is close to a classical real 2-D. Wavelet transform at each scale in one tree, and the discrete transform can be implemented by a ladder filter structure[3]. It can reserve the needed details or the approximation of a given scale, while remove all other scales, and shift the input image to produce a shift of the reconstructured filtered image without aliasing. The superiority of DT-CWT is that it can separete more directions than the real wavelet transfom. The 2-D DWT produces 3 band-pass subimages at each level, while the 2-D DT-CWT can provide 6 band-pass 
subimages in two adjacent spectral quadrants at each level, so the positive frequencies can be separete from negative ones vertically and horizontally.Figure 2 shows the region-based fushion method for infrared and virsual images adapts the DT-CWT for its nearly shift invariant and limited redundancy. The diagram of the proposed region-based method with DT-CWT as Figure1 showing:

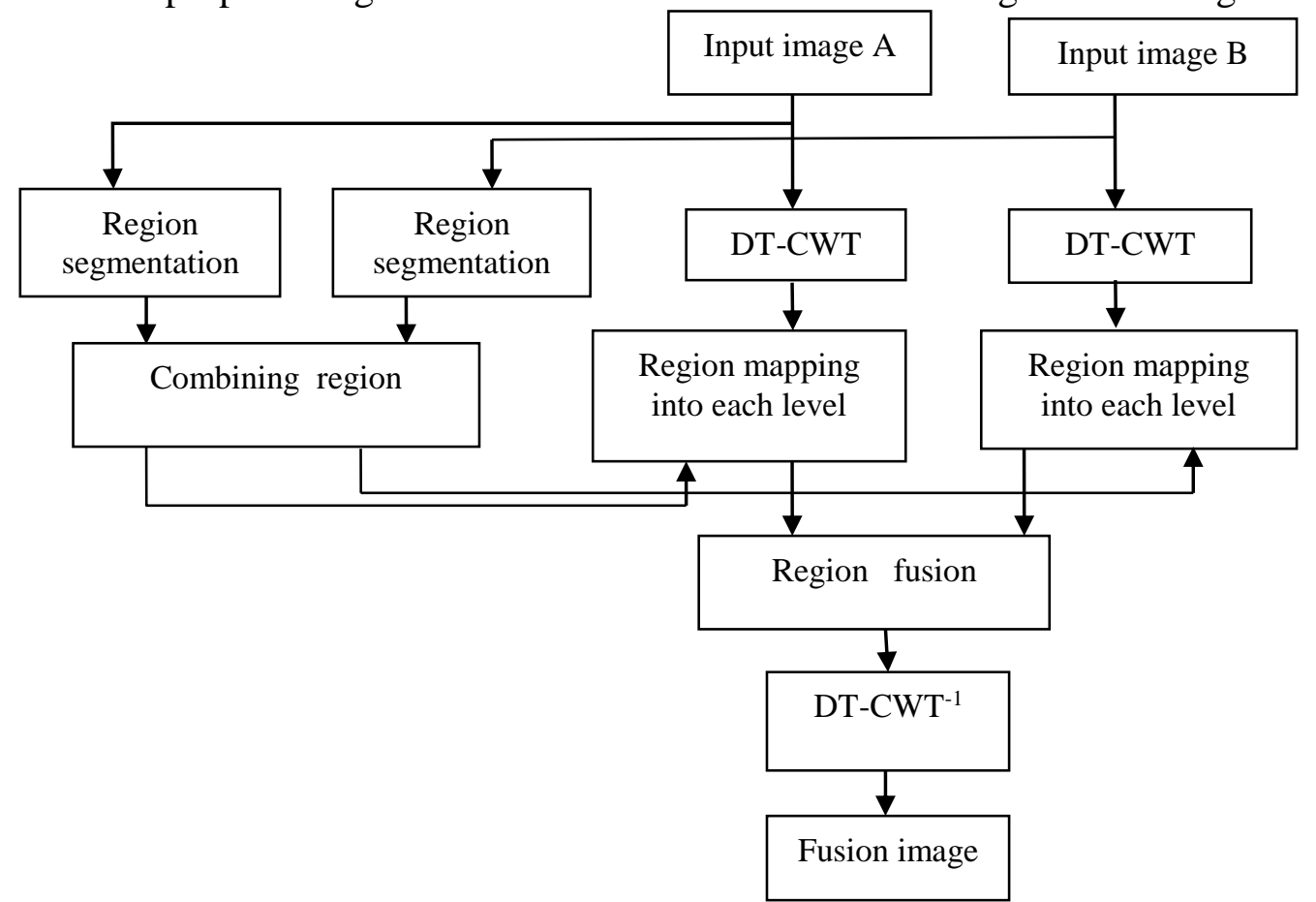

Figure 1 The diagram of the proposed region-based method with DT-CWT

\subsection{Image fusion based on region segmentation and complex wavelet}

As Figure1showing, Segmentation is firstly performed on the low level visible light image marked A and infrared image marked B respectively, consequently, the DT-CWT coefficients from the different regions are merged separately, finally the fused image is obtained by operating inverse DT-CWT.

Decomposed by the multi-resolution DT-CWT, low-frequency part of the images denotes their approximate components, which contains spectral information of the source image. High-frequency part of the images denotes their detail components, which contains edge detail information of the source image.Usually the fusion rules are commonly that average operator is used in low frequency domain, and max absolute operator is used in high frequency domain.For the two fused source images of the same scene, spectral information of visible light image is much richer than the infrared image. When the fusion of average operator is adopted, part of spectrum information of visible light image will be lost. In order to settle this problem, we adopt spatial frequency to guide region-based fusion.The spatial frequency has led to an effective objective quality index for image fusion.

\subsection{Image fusion experiment}

An experiment has been done in the method of the above, and the result showed in Figure 2. The infrared and visible image of the same scene are fused by region-based method with DT-CWT. Meanwhile region-based colour fusion is used, that is the image segmetation is firstly carried out, then region colouring is realized. Its major points are (a) the infrared and visible images are rendered segment-by-segment; (b) the segmented regions of the two images are combined and form a new segmented map; (c) the regions are classified according to the target types and the spatial frequencies. According to the fusion images, this method has good effectiveness, which preserves not only the spectral information of the visible light image, but also the thermal target information of the infrared image. According the fused image in Figure2, it should be noted that both the spatial and spectrial resolutions have been enhenced, in comparision to the original images. The spectial information has been increased and the structural information in the original multispectrial image has also been enriched, so the fused image has a better visual effect. 


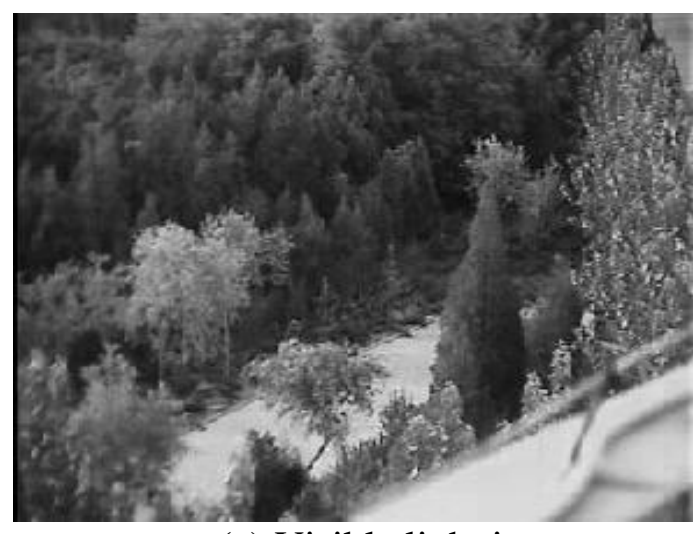

(a) Visible light image

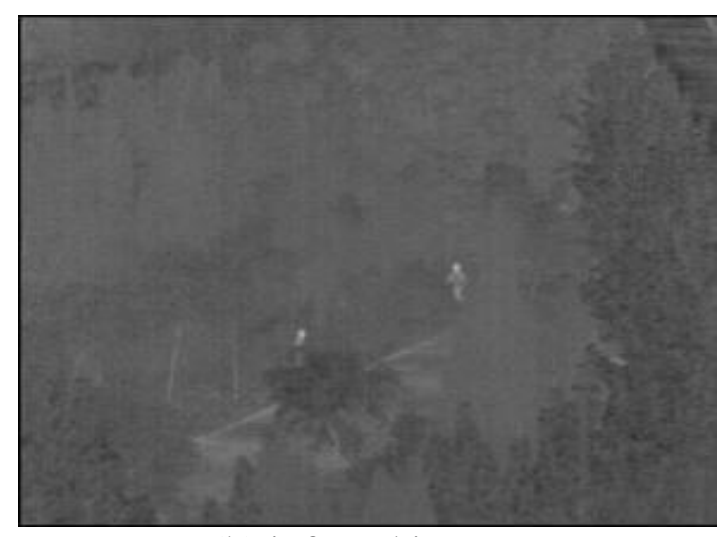

(b) infrared image

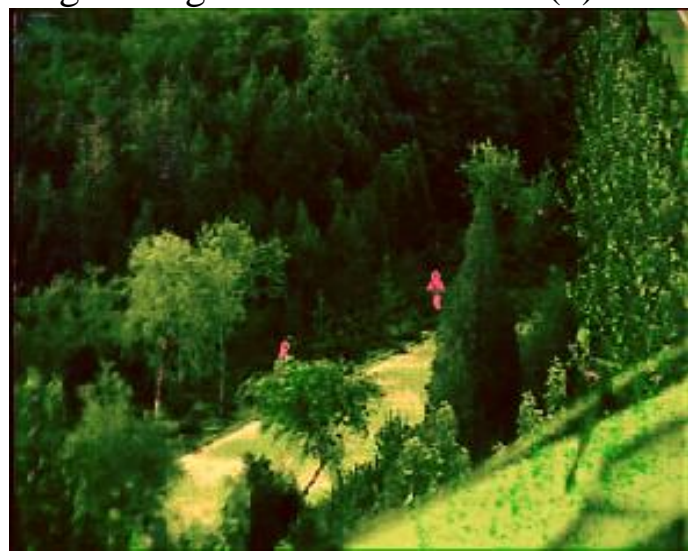

(c)Fused image

Figure 2. Visible light image and infrared image fused by region-based method with DT-CWT

Since the human eye can discern several thousand colours, while it can only distinguish about 100 shades of gray in any case, so mapping multiple spectral bands of imagery into a 3-D colour space is presented,which increase the dynamic range of a sensor system.Experiments have convincing demostrated that appropriately designed false colours rendering of imagery can significantly improve observer performance and reaction times in tasks that involve scene segmentation and classification.However inappropriate colour mappings may hinder situational awareness ,as Figure 3(a) Showing. A solution to this problem is ergonomic colour scheme that can produce imagery with natural appearance and with colours invariant for change in the environmental conditons, as Figure 3(c) Showing. Compared Figure 3(a) and (c), it is clear that in Figure 3(c) the counter intuitive appearance of the scene is more legible.

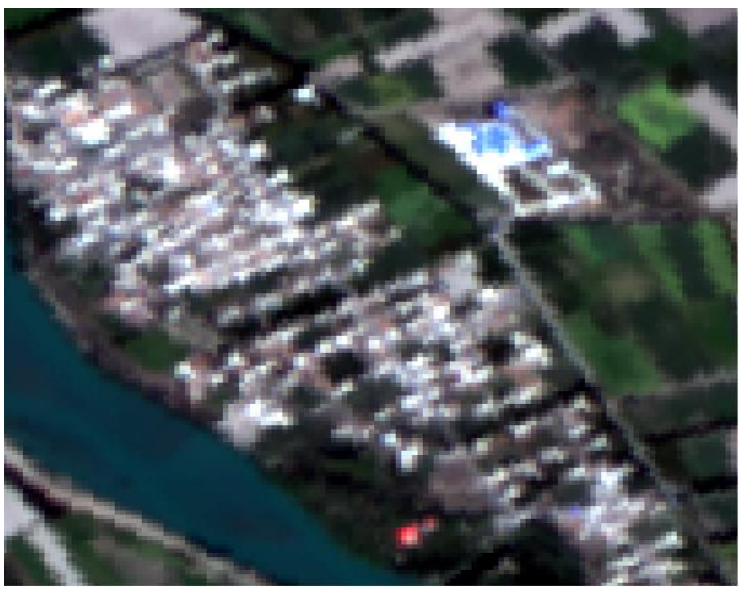

(a) 3-D colour space mapping by false colour

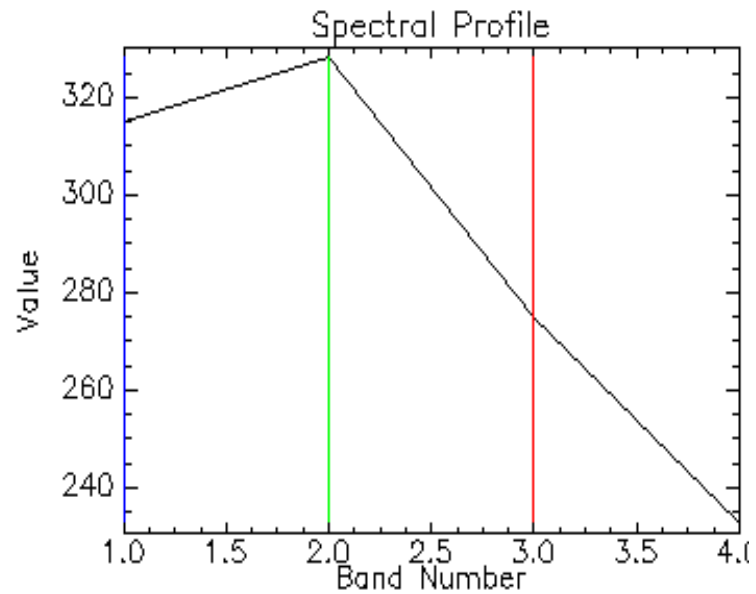

(b) The mapping multiple spectral bands of imagery 


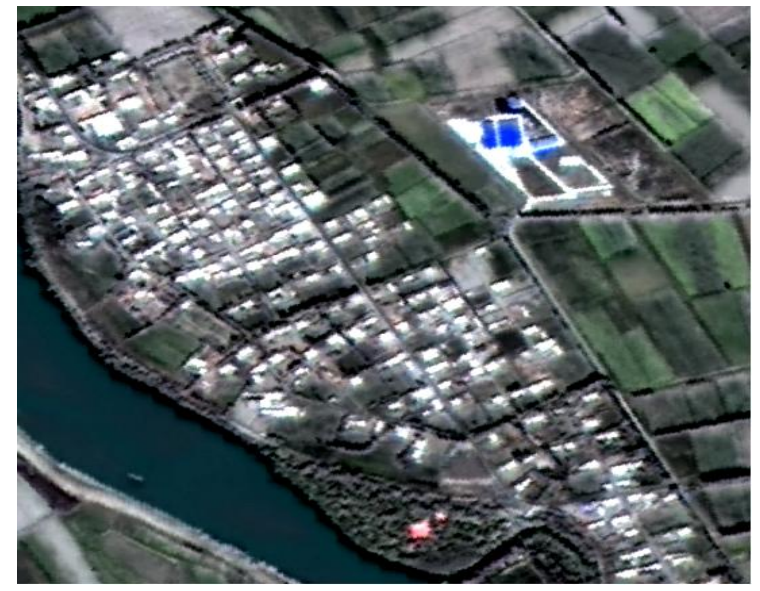

(c) Image fusion with ergonomic colour scheme

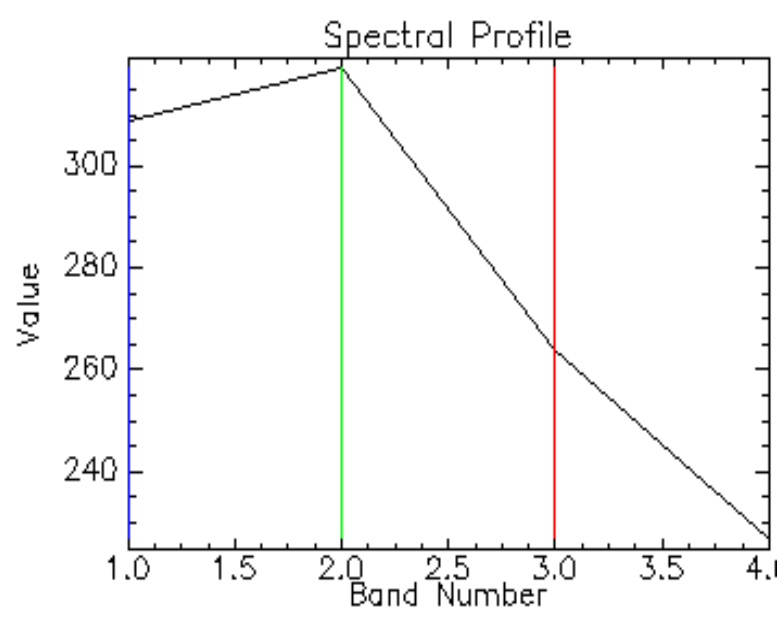

(d) Ergonomic colour scheme bands

Figure3. Image fusion with 3-D colour space mapping and ergonomic colour scheme

\section{Conclusion}

The proposed method which uses Dual-Tree Complex Wavelet Transform and region-based method is applied to fuse the infrared and visible image of the same scene, both the spatial and spectrial resolutions have been enhenced, in comparision to the original images. Dual-Tree Complex Wavelet Transform has the following propeties: approximate shift invariance, good directional selectivity in 2-D with Gabor-like filter also true for higher dimensionality: perfect reconstruction using short linear-phase-filter.Advances in night vision imaging have provided alternative methods since signals from image intensifier and uncooled infrared sensors may be combined with in a single image format,and the spectrum may be efficciently combined.In order to be more convenient for human eyes, the 3-D colour space is presented and some improvement has been done that is ergonomic colour scheme, which can produce imagery with natural appearance and with colours invariant for change in the environmental conditons. This make the counter intuitive appearance of scenes more legible.

\section{Acknowledgments}

This work was financially supported by the National Natural Science Foundation of China (Grant No.61307002, 61275184), the National Major Project (Grant No. 32-Y30B08-9001-13/15), the Natural Science Foundation of Shaanxi Province (No.2012JM1009), the Scientific Research Program of Shaanxi Provincial Education Department (No.12JK0984), the Scientific Research Program (No.2XSYK017, No.12XSYK014) and the Teaching Reform Research Program of Xianyang Normal University (No. 201200127), China.

\section{References}

[1] K. Amolins, Y. Zhang, and D. Peter, "Wavelet based image fusion techniques-an introduction, review and comparision,” J. Photogram. \& remote sening. 2007, vol. 62, pp. 249-263.

[2] Gatos B, Pratikakis I, Perantonis S J. Adaptive degraded document image binariza-tion [J]. Pattern Recognition, 2006, 39(3):317--327.

[3] GonzalezR.C, WoodsR.E. DigitalImageProcessing [M]. NewJersey, USA: New Jersey:Prentice Hall, 2002

[4] Dabov K, Foi R, Katkovnik V, et al. BM3D image denoising with shape-adaptive principal component analysis [C]Proc. Workshop on Signal Processing with Adaptive Sparse Structured Representations (SPARS). Saint-Malo, France: INRIA, 2009. 
[5] Dabov K, Foi R, Katkovnik V, et al. Image restoration by sparse 3D transform-domain collaborative filtering [C]SPIE Electronic Imaging. Bern, Switzerland: SPIE, 2008

[6] KervrannC,BoulangerJ. Optimalspatialadaptationforpatchbase dimage denoising [J]. IEEE Transactions on Image Processing, 2006, 15(10): 2866--2878.

[7] Kervrann C, Boulanger J. Local adaptivity to variable smoothness for exemplar-based image regularization and representation [J]. International Journal of Com-puter Vision, 2008, 79 (1): 45--69

[8] Pai Y, Chang Y, Ruan S. Adaptive thresholding algorithm: Efficient computation technique based on intelligent block detection for degraded document images [J]. Pattern Recognition, 2010, 43(9): 3177--3187

[9] LuChen Guangyi, Qian Shen-En. Denoising of Hyperspectral Imagery Using Principal Component Analysis and Wavelet Shrinkage, IEEE Transactions on Geoscience and Remote Sensing, 2011, 49 (3): 973 980. 